\title{
Proposed Revision of the Conventional Method of Wave-Filter Design
}

\author{
Paul J. Selgin
}

\begin{abstract}
A modification of the conventional "classical" method of electrical filter design is proposed. The change consists of the use of new parameters, "frequency numbers", which permit the design to be made from specification of peak and cut-off attenuation rather than idealized cut-off frequency, as in the conventional method.

The method is extended to cover the dissipative, as well as the nondissipative, or lossless case. Suitable design curves and tabulations are included.
\end{abstract}

\section{Introduction}

The so-called conventional method of wave-filter design is not as powerful nor as flexible as more recent methods, based on the "lattice" form of filter section $\lceil 1] ; 1^{1}$ nevertheless, it still has usefulness because of its relative simplicity, and is, in fact, used by practically all designers of equipment to whom the solution of a filter problem is an occasional necessity rather than a constant challenge.

Because simplicity is the main virtue of the conventional approach, one is led to ask whether the ultimate of simplicity and effectiveness has been reached in this particular domain, or otherwise. Upon examining the question, one finds that the conventional method, since its inception (Zobel's first paper on the subject [2] was published in 1923) has remained essentially unchanged, the attention of network specialists having been diverted, by the work of Cauer [3] and Bode [4], to other methods of synthesis.

The chief purpose of this paper is to make the conventional image method better suited to the solution of practical filter problems, having regard to the form in which the design data must be formulated. In practice the cut-off frequency as such is not given, but minimum and maximum attenuations are given together with the respective frequency ranges (see fig. 7). The present design method is geared to this formulation of the problem and does not require assumptions regarding the cut-off frequency value. Also, the method permits the minimizing of reflection losses at the critical frequencies by removing the arbitrary assumption that the "nominal" filter impedance must coincide with the load impedance.

The method is further refined by suitably selecting the parameters that determine the values to be used in the elements of each filter section. In the classical method the cut-off frequency is one such parameter, the cut-off attenuation another. In the present approach a single function of frequency, the "frequency number," is used throughout, and values of this function corresponding to the cut-off and peakattenuation frequencies are used as the design parameters.

\footnotetext{
1 Figures in brackets indicate the literature references at the end of this pape:.
}

\section{Basic Structures}

Space does not permit a full treatment of all filter types in this article. We will, therefore, limit the discussion to the three most frequently used types: high-pass, low-pass, and symmetrical band-pass.

The basic " $L$ " structure, or "half section," foi" these three classes is shown in figure 1, in two equivalent configurations, su:table for the formation of " $T$ " section and " $\pi$ " sections, respectively. The so-called "T-connected" configuration will be given particular attention in the discussion. For each class is given, along with the two alternative configurations, the "frequency number" that is, the function of frequency that should be used, instead of the frequency itself, in all compuations concerning the filter.

\section{Dependence of the Transfer Constant on the Arms Ratio}

The ratio $Z_{s} / Z_{p}$ between the series arm and shunt arm impedances of the $L$ half section determines the attenuation and phase shift characteristics of the image-terminated $T$ or $\pi$ section obtained by joining two such half sections. (Conventional filter theory is predicated on the existence of image-termination or "image match" [5] between the various sections and to the remainder of the system.) The complex number $\alpha+j \beta$, where $\alpha$ is the attenuation in nepers, $\beta$ the phase shift in radians, is, in general, the transfer constant $\theta$ of an image-connected 4-pole. According to this definition, $\theta$ may be shown to equal the complex ratio of output to input voltage (output to input current) of the 4-pole [5, p. 30].

In the following, $\theta$ will always be associated to a complete $T$ or $\pi$ section (it makes no difference which), while $Z_{s}$ and $Z_{p}$ will be the values for the component half-sections.

The relationship between $Z_{s} / Z_{p}$ and $\theta$ is the following:

or, expressing $e^{\theta}$ :

$$
\frac{Z_{s}}{Z_{p}}=\sinh ^{2} \frac{\theta}{2}
$$

$$
e^{\theta}=\frac{\sqrt{1+\frac{Z_{s}}{Z_{p}}}+\sqrt{\frac{Z_{s}}{Z_{p}}}}{\sqrt{1+\frac{Z_{s}}{Z_{p}}}-\sqrt{\frac{Z_{s}}{Z_{p}}}} .
$$



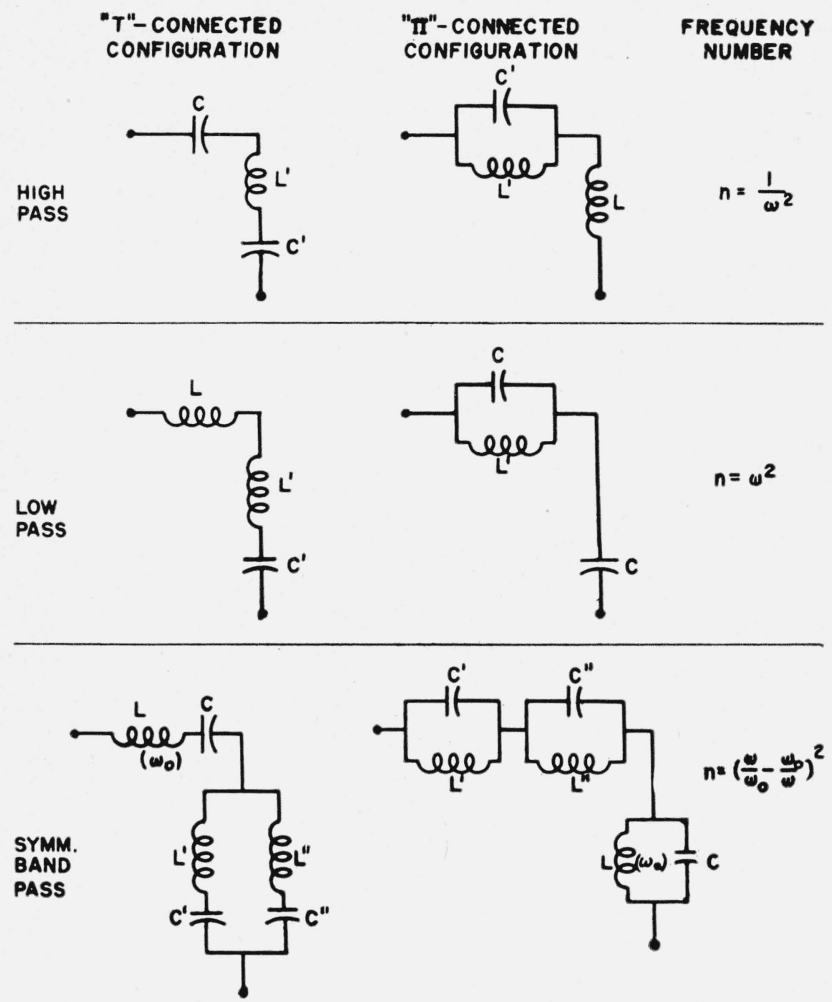

Figure 1. Basic "L" half-sections.

These expressions and the corresponding families of curves will be found in any standard textbook on filters $[6] .^{2}$

The dependence of $\alpha$ and $\beta$ on $Z_{s} / Z_{p}$, for $Z_{s}$ and $Z_{p}$ purely reactive, may be summarized in the diagram of figure 2, which is schematic and not suitable for purposes of evaluation.

\section{Arms Ratio Expressions}

From what has been said it is apparent that the arms ratio $Z_{s} / Z_{p}$ determines the transmission characteristics of the filter section. At the moment we are interested only in the dissipation-less case; however, since ultimately dissipation will have to be considered, it is convenient (in spite of the added algebraic difficulty) to derive at the outset the arms ratio

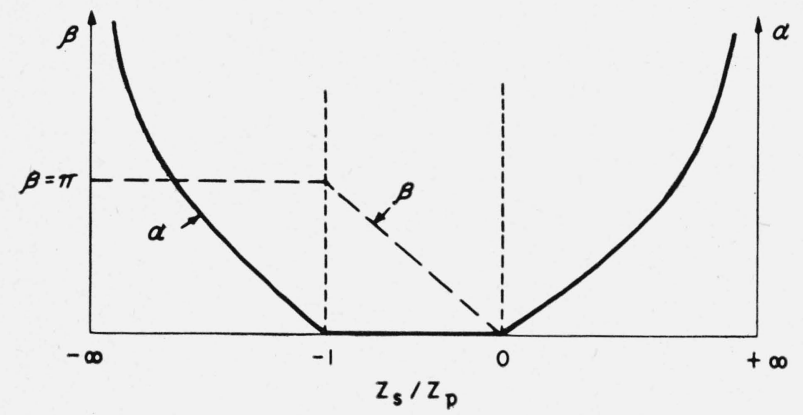

Figure 2.

${ }^{2}$ A set of curves of constant $U$ and $V$ (real and imagina:y parts of $Z_{s} / Z_{p}$ in the $\alpha, \boldsymbol{\beta}$ plane), is given on figure 8 .

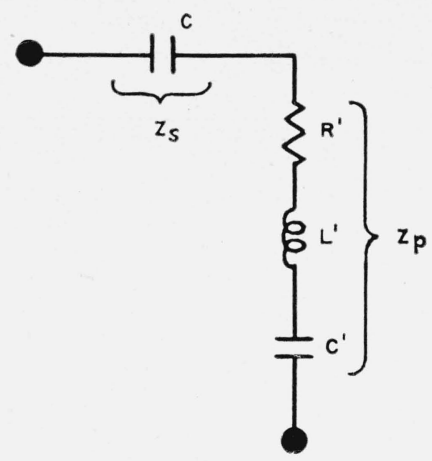

Figure 3.

for the case of dissipative structures, differing from those of figure 1 in that the pure inductances are replaced by physical coils of given dissipation factor $d=R / \omega L$, approximately constant over the operating range. The three classes of figure 1 will be considered in turn.

\subsection{High-pass}

Consider the first form (for $T$ connection). The equivalent circuit, allowing for dissipation in the coil, is shown in figure 3 . The arms ratio is:

$$
\begin{aligned}
\frac{Z_{s}}{Z_{p}} & =\frac{1}{j C \omega\left(R^{\prime}+j \omega L^{\prime}+\frac{1}{j \omega C^{\prime}}\right)} \\
& =\frac{-1}{C L^{\prime} \omega^{2}\left(1-j d-\frac{1}{L^{\prime} C^{\prime} \omega^{2}}\right)}
\end{aligned}
$$

At this point we discard $\omega$ as the variable denoting frequency and use instead the frequency number appropriate for this class of filters, that is (fig. 1) the variable:

$$
n=\frac{1}{\omega^{2}}
$$

Equation 3 can be rewritten in terms of $n$ by noting that for zero dissipation, the infinite attenuation frequency is given by

$$
n=n_{\infty}=\frac{1}{\omega_{\infty}^{2}}=L^{\prime} C^{\prime}
$$

and the lower cut-off frequency $\left(Z_{s} / Z_{p}=-1\right.$ or $\left.n=n_{c}\right)$ by

$$
\frac{n_{c}}{L^{\prime} C\left(1-\frac{n_{c}}{n_{\infty}}\right)}=1 .
$$

These relations allow (3) to be written as

$$
\frac{Z_{s}}{Z_{p}}=\left(\frac{n}{n_{\infty}}-\frac{n}{n_{c}}\right) \frac{1-\frac{n}{n_{\infty}}+j d}{\left(1-\frac{n}{n_{\infty}}\right)^{2}+d^{2}} .
$$


Since the dissipation factor $d$ (reciprocal of the coil $Q$ ) is always a small number, it is permissible to neglect $d^{2}$ in the above, provided we do not attempt to use the resulting expressions in the immediate neighborhood of the frequency of peak attenuation, in which case the denominator of (7) becomes infinite if $d^{2}$ is neglected. This is not a serious limitation to the design of dissipative filters, since the peak values of attenuation are never critical, being far in excess of design limits.

With this simplification, (7) goes into the form:

$$
\frac{Z_{s}}{Z_{p}}=\frac{\frac{n}{n_{\infty}}-\frac{n}{n_{c}}}{1-\frac{n}{n_{\infty}}}\left(1+j d \frac{1}{1-\frac{n}{n_{\infty}}}\right) .
$$

The same expression goes for the second alternative form of high-pass filter section, which is in dual relationship to the first (7).

\subsection{Low-Pass}

Again consider the $T$ connected form (fig. 1). The equivalent circuit for dissipative coils appears in figure 4.

The arms ratio is:

$$
\frac{Z_{s}}{Z_{p}}=\frac{j \omega L(1-j d)}{j \omega L^{\prime}\left(1-j d-\frac{1}{L^{\prime} C^{\prime} \omega^{2}}\right)} .
$$

Discarding $\omega$ in favor of the frequency number $n$, as before, we let:

$$
n=\omega^{2}
$$

Again, in the dissipation-less case we have

$$
n_{\infty}=\omega_{\infty}^{2}=\frac{1}{L^{\prime} C^{\prime}}
$$

and at cut-off:

$$
-1=\frac{L}{L^{\prime}} \frac{1}{1-\frac{n_{\infty}}{n_{c}}}
$$

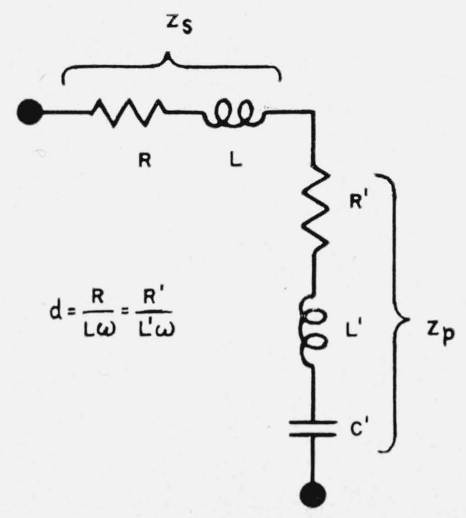

Figure 4. so that (9) becomes

$$
\frac{Z_{s}}{Z_{p}}=\left(\frac{n_{\infty}}{n_{c}}-1\right) \frac{1-\frac{n_{\infty}}{n}+d^{2}+j d \frac{n_{\infty}}{n}}{\left(1-\frac{n_{\infty}}{n}\right)^{2}+d^{2}}
$$

As in the high-pass case, we may neglect $d^{2}$ with the understanding that the resulting expressions will not be accurate in the neighborhood of the peak attenuation frequency. Hence:

$$
\frac{z_{s}}{z_{p}}=\frac{\frac{n}{n_{\infty}}-\frac{n}{n_{c}}}{1-\frac{n}{n_{\infty}}}\left(1-j d \frac{1}{1-\frac{n}{n_{\infty}}}\right)
$$

The dual low-pass form, for $\pi$ connection, yields the same value for $Z_{s} / Z_{p}$.

\subsection{Symmetrical Band-Pass}

The impedance of a series resonant arm,

$$
Z=R+j \omega L+\frac{1}{j \omega c}
$$

may conveniently be written

$$
Z=r(d+j W)
$$

where

$$
\left.\begin{array}{rl}
r & =\sqrt{\frac{L}{C}} \\
d & =R \sqrt{\frac{C}{L}} \\
W & =\frac{\omega}{\omega_{0}}-\frac{\omega_{0}}{\omega} \\
\omega_{0} & =\frac{1}{\sqrt{L C}}
\end{array}\right\}
$$

Using this expression for the series combinations of the band-pass structure (fig. 5), its arms ratio takes the form:

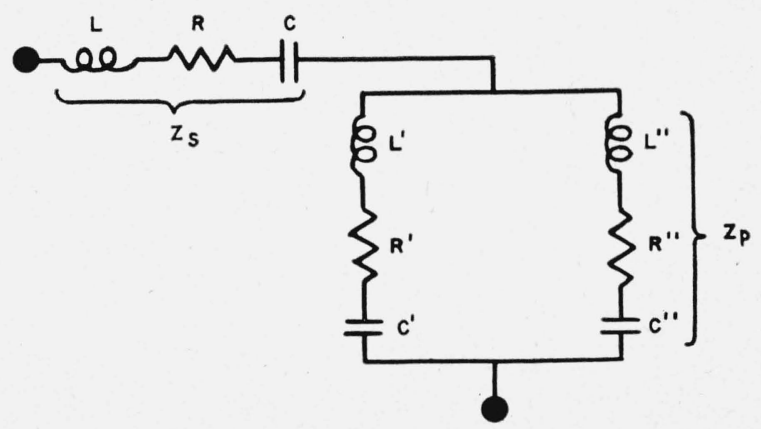

Figcre 5 . 


$$
\frac{Z_{s}}{Z_{p}}=r(d+j W)\left[\frac{1}{r^{\prime}\left(d^{\prime}+j W^{\prime}\right)}+\frac{1}{r^{\prime \prime}\left(d^{\prime \prime}+j W^{\prime \prime}\right)}\right]
$$

Design requirements inherent to the band-pass filter impose a definite relationship upon the coefficients. This is best understood by examing the behavior of the function $Z_{s} / Z_{p}$ with frequency in the nondissipative case.

In the dissipation-less case,

and

$$
\frac{Z_{s}}{Z_{p}}=0 \text { when } W=0, \text { at } \omega=\omega_{0}=\frac{1}{\sqrt{L C}}
$$

$$
\begin{aligned}
\frac{Z_{s}}{Z_{p}}= & \infty \text { when } W^{\prime} \text { or } W^{\prime \prime}=0, \text { at } \omega_{\infty}^{\prime}=\frac{1}{\sqrt{L^{\prime} C^{\prime}}} \text { or } \omega_{\infty}^{\prime \prime}= \\
& \frac{1}{\sqrt{L^{\prime \prime} C^{\prime \prime}}} \text {, respectively. }
\end{aligned}
$$

In general, the band-pass structure yields two passbands. To make these contiguous, we must meet the "condition of congruence" [8] that $r W$ and $1 / r^{\prime} W^{\prime}+1 / r^{\prime \prime} W^{\prime \prime}$ vanish at a common frequency. Since $W=0$ at $\omega=\omega_{0}$ we need

$$
\frac{1}{r^{\prime}\left(\frac{\omega_{0}}{\omega_{\infty}^{\prime}}-\frac{\omega_{\infty}^{\prime}}{\omega_{0}}\right)}+\frac{1}{r^{\prime \prime}\left(\frac{\omega_{0}}{\omega_{\infty}^{\prime \prime}}-\frac{\omega_{\infty}^{\prime \prime}}{\omega_{0}}\right)}=0
$$

If the filter is to have a symmetrical pass characteristic (on a logarithmic frequency scale),

leading directly to

$$
\frac{\omega_{0}}{\omega_{\infty}^{\prime}}=\frac{\omega_{\infty}^{\prime \prime}}{\omega_{0}}
$$

$$
r^{\prime}=r^{\prime \prime}
$$

If we add the condition that each series resonant arm has the same dissipation factor $d,(17)$ simplifies to

$$
\frac{z_{s}}{z_{p}}=\frac{r}{r^{\prime}}(d+j W)\left[\frac{\mathrm{I}}{d+j W^{\prime}}+\frac{1}{d+j W^{\prime \prime}}\right] .
$$

As before, the denominator is to be rationalized and $d^{2}$ neglected, yielding

$$
\frac{z_{s}}{z_{p}}=\frac{r}{r^{\prime}} \frac{W\left(W^{\prime}+W^{\prime \prime}\right)}{W^{\prime} W^{\prime \prime}}\left\{1+j d\left[\frac{W^{\prime 2}+W^{\prime 2}}{W^{\prime} W^{\prime \prime}\left(W^{\prime}+W^{\prime \prime}\right)}-\frac{1}{W}\right]\right\} \text {. }
$$

We now define the frequency number for the symmetrical band-pass case as

$$
n=\left(\frac{\omega}{\omega_{0}}-\frac{\omega_{0}}{\omega}\right)^{2}=W^{2}
$$

for a generic value of frequency; hence the value:

$$
n_{\infty}=\left(\frac{\omega_{\infty}^{\prime}}{\omega_{0}}-\frac{\omega_{0}}{\omega_{\infty}^{\prime}}\right)^{2}=\left(\frac{\omega_{\infty}^{\prime \prime}}{\omega_{0}}-\frac{\omega_{0}}{\omega_{\infty}^{\prime \prime}}\right)
$$

for either frequency of peak attenuation, by (19), and the value:

$$
n_{c}=\left(\frac{\omega_{c}^{\prime}}{\omega_{0}}-\frac{\omega_{0}}{\omega_{c}^{\prime}}\right)^{2}=\left(\frac{\omega_{c}^{\prime \prime}}{\omega_{0}}-\frac{\omega_{0}}{\omega_{c}^{\prime \prime}}\right)^{2}
$$

for either cut-off frequency (again because of symmetry).

Equation 22 may now be rewritten in terms of the above values of $n$ in place of $W, W^{\prime}, W^{\prime \prime}$. By using the identities:

$$
\left\{\begin{array}{l}
W=\sqrt{n} \\
W^{\prime}+W^{\prime \prime}=\sqrt{n} \sqrt{n_{\infty}+4} \\
W^{\prime} W^{\prime \prime}=n-n_{\infty} \\
W^{\prime 2}+W^{\prime \prime 2}=n n_{\infty}+2\left(n+n_{\infty}\right)
\end{array}\right\}
$$

we find:

$\frac{Z_{s}}{Z_{p}}=\frac{r}{r^{\prime}} \frac{n \sqrt{n_{\infty}+4}}{n-n_{\infty}}\left\{1+j \frac{d}{\sqrt{n}}\left[\frac{n n_{\infty}+2\left(n+n_{\infty}\right)}{\left(n-n_{\infty}\right) \sqrt{n_{\infty}+4}}-1\right]\right\}$

We have not yet imposed the condition that $Z_{s} / Z_{p}$ must equal -1 (for $d=0$ ) at the cut-off frequencies, corresponding to $n=n_{c}$. There is no ambiguity on this point, because the other possible value that $Z_{s} / Z_{p}$ might take at the cut-off frequencies, which is zero, actually occurs at the midband frequency, $\omega_{0}$, the cut-off frequency common to two pass-bands, which merge into one by virtue of the "congruence" condition.

The cut-off relation is

$$
-1=\frac{r}{r^{\prime}} \frac{n_{c} \sqrt{n_{\infty}+4}}{n_{c}-n_{\infty}},
$$

and the arms ratio becomes:

$$
\frac{Z_{s}}{Z_{p}}=\frac{\frac{n}{n_{\infty}}-\frac{n}{n_{c}}}{1-\frac{n}{n_{\infty}}}\left\{1+\frac{j d}{\sqrt{\eta}}\left[\frac{n n_{\infty}+2\left(n+n_{\infty}\right)}{\left(n-n_{\infty}\right) \sqrt{n_{\infty}+4}}-1\right]\right\}
$$

\section{Determination of the Section Numbers and of the Cut-Off Frequency}

The real part of the ratio $Z_{s} / Z_{p}$ is independent of $d$ and is given by the same expression for all three cases (8), (14), (29):

$$
\operatorname{Rc}\left(\frac{Z_{s}}{Z_{p}}\right)=\left(\frac{Z_{s}}{Z_{p}}\right)_{d=0}=\frac{X_{s}}{X_{p}}=\frac{\frac{n}{n_{\infty}}-\frac{n}{n_{c}}}{1-\frac{n}{n_{\infty}}},
$$

where $n$ is the "frequency number" appropriate to each case in turn. 
We may now substitute this value in eq 2 and thereby obtain expressions suitable for the construction of universal sets of curves of attenuation and phase shift, valid for all the filter sections shown in figure 1. To avoid imaginary arguments, three distinct cases (corresponding to the three ranges of fig. 2) will be considered:

(1):

$(2)$ :

$$
\alpha=0, \beta \neq 0,0>\frac{X_{s}}{X_{p}}>-1, \quad n<n_{c}<n_{\infty}
$$

(transmitted frequency range)

$$
\beta=2 \sin ^{-1} \sqrt{-\frac{X_{s}}{X_{p}}}=2 \sin ^{-1} \sqrt{\frac{\frac{n_{c}}{n_{\infty}}-1}{\frac{n_{c}}{n}-\frac{n_{c}}{n_{\infty}}}} .
$$

(3):

$$
\begin{array}{r}
\alpha \neq 0, \beta=0, \frac{X_{s}}{X_{p}}<0, n_{c}<n_{\infty}<n \rightarrow \\
\alpha=2 \sinh ^{-1} \sqrt{\frac{X_{s}}{X_{p}}}=2 \sinh ^{-1} \sqrt{\frac{1-\frac{n_{c}}{n_{\infty}}}{\frac{n_{c}}{n}-\frac{n_{c}}{n_{\infty}}}} .
\end{array}
$$

$$
\alpha \neq 0, \beta= \pm \pi \frac{X_{s}}{X_{p}}<-1, n_{c}<n<n_{\infty}
$$

$$
\alpha=2 \cosh ^{-1} \sqrt{\frac{X_{s}}{X_{p}}}=2 \cosh ^{-1} \sqrt{\frac{1-\frac{n_{c}}{n_{\infty}}}{\frac{n_{c}}{n}-\frac{n_{c}}{n_{\infty}}}} .
$$

Note that in all cases $n_{\infty}>n_{c}$. The value of $\alpha$ or $\beta$ is seen to depend on two ratios, $n_{c} / n$ and $n_{c} / n_{\infty}$. For a given ratio $n_{c} / n_{\infty}, \alpha$ or $\beta$ may be plotted against $n_{c} / n$. These plots may be used to select values of the ratio $n_{c} / n_{\infty}$, which we will call the section number and designate by $N$ :

$$
N=\frac{n_{c}}{n_{\infty}} .
$$

If more than one section is used in a filter, a different value of $N$ will generally be used for each section. As a rule there will be one section having $N=0$ (the "prototype" of the literature) and one section having $0.6<N<0.7$ : the latter is usually divided into two half sections which serve as terminations for the filter.

Another purpose of the attenuation plots (fig. 6) is the determination of the cut-off frequency (or frequencies). This is generally treated as a known requirement of the filter, but actually it is not. What is required of a filter may be summarized in figure 7, which is drawn for the high-pass case. Two frequencies and two values of attenuation are assigned: the pass band is assigned by a "boundary" value of frequency, $f_{a}$, and a value of permissible attenuation, $a$; the attenuation band, by a boundary frequency $f_{b}$ and a required attenuation, $A$. The theoretical cut-off frequency generally lies somewhere between the two boundaries. To determine its exact location, the section number for the entire filter must be tentatively established. Suppose for example a three-section high-pass filter were to be based on the requirements of figure 7 . Let us tentatively make:

$$
\begin{aligned}
& N=0 \quad \text { for section } 1 \\
& N=0.5 \text { for section } 2 \\
& N=0.7 \text { for section } 3
\end{aligned}
$$

Adding the attenuations plotted on figure 6 for the above values of $N$ over a range of values of $n_{c} / n$, we find that the over-all attenuation goes through the value $55 d b$ (the value of $A_{1}$ required attenuation) when $n_{c} / n$ has the value 0.74 . We may

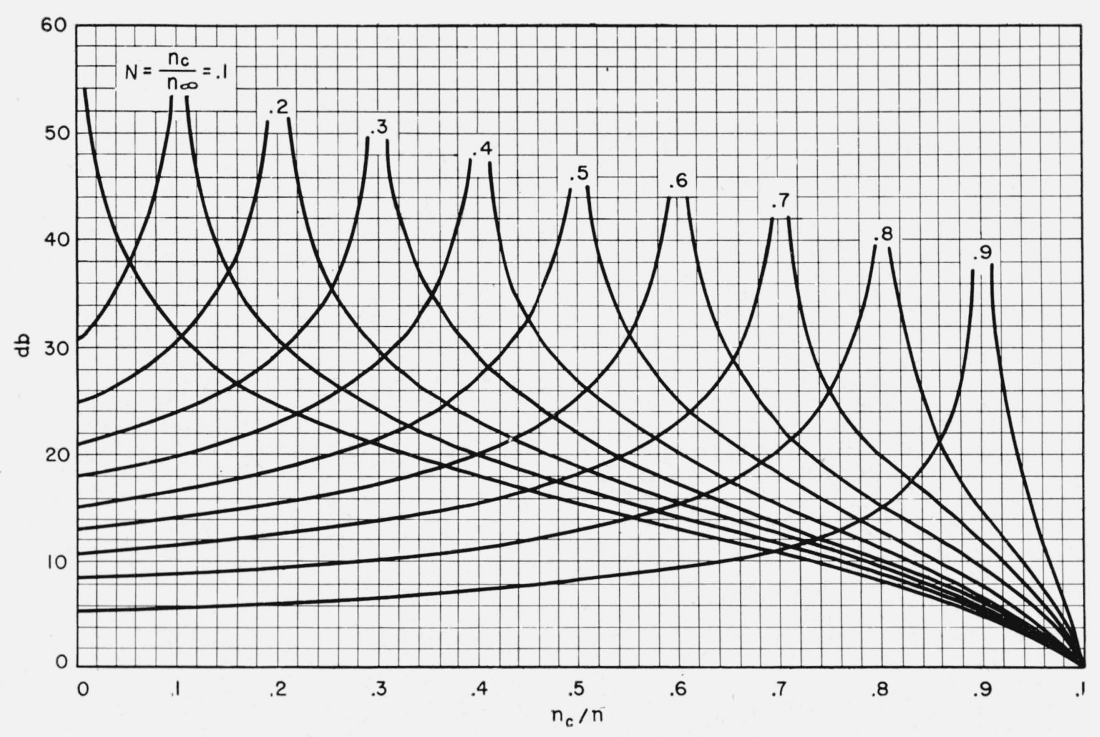

Figure 6. Plot of $\alpha$ against $n_{c} / n$ for various values of $n_{c} / n \infty$. 


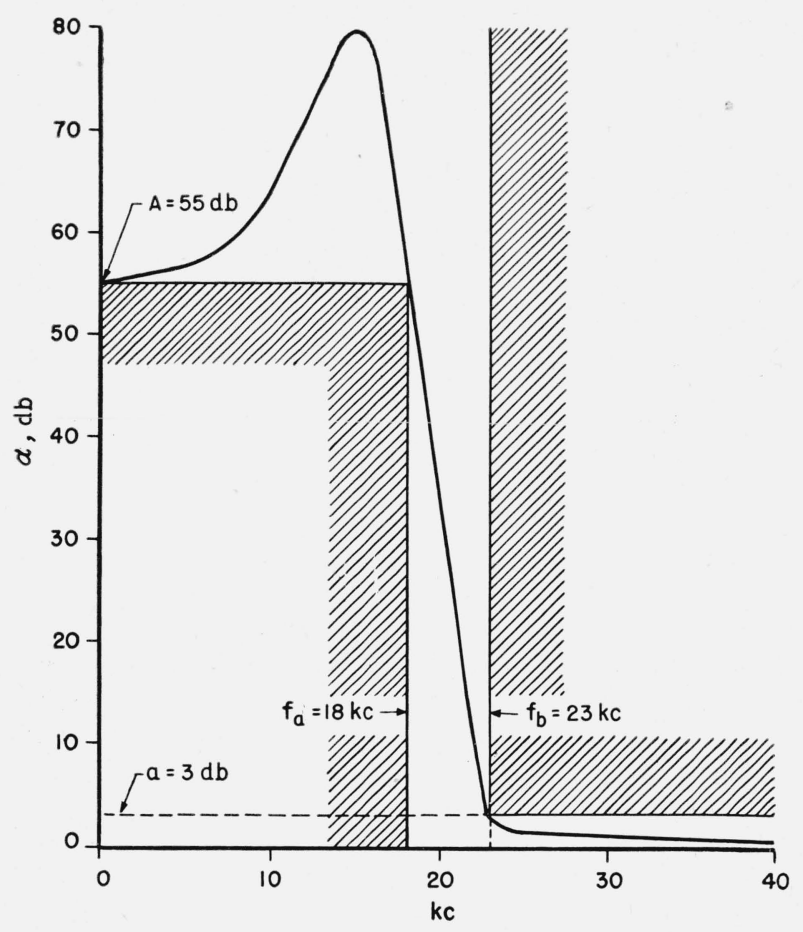

Figure 7.

therefore impose the condition $n=n_{a}$ for $n_{c} / n=0.74$, thus causing the attenuation to be exactly equal to $A$ at the "boundary frequency" $f_{a}$ (fig. 7). Hence we write:

$$
\frac{n_{c}}{n_{a}}=0.74
$$

from which recalling the equivalence $n=1 / \omega^{2}$ for high-pass filters:

$f_{c}=f_{a} \frac{1}{\sqrt{0.74}}=20.9 k c ; n_{c}=\frac{1}{\left(2 \pi f_{c}\right)^{2}}=5.8 \times 10^{-6}$

The selection of the section number may be based on several criteria. If the problem is given in the terms of figure 7 , the designer will attempt to secure the necessary minimum attenuation $A$ (in the example $55 d b)$ with the least number of sections. Charts that permit the automatic selection of the optimum section number, and give at the same time the value $n_{c} / n_{a}$, for filters of three sections or less, have been published by the writer [7] on the basis of this criterion.

Another possible consideration affecting the selection of the section number is the phase-shift over the transmitted band. It may be desirable to have the phase shift vary as linearly as possible with the frequency, to eliminate phase distortion. Generally speaking, a certain amount of "cut and try" is unavoidable when designing a filter by the conventional method. The labor involved may be minimized, however, by a favorable choice of parameters, which justifies the present treatment.

\section{Determination of the Maximum Permis- sible Dissipation Factor}

Given the values of $N=n_{c} / n_{\infty}$ for the different sections, we may now determine for each value of $n$ (that is, for each frequency), $Z_{s} / Z_{p}$ in the dissipative case $(d \neq 0)$ using one of the three equations (8), (14) and (29) (fig. 10 may conveniently be used in place of eq. 29). From the values of $Z_{s} / Z_{p}$, using eq 2 or an appropriate chart (fig. 8), the true values of $\alpha$ and $\beta$, may be obtained.

It is not necessary, as a rule, to obtain these values for more than one or two values of frequency. A check on the attenuation at the edge of the transmitted band (frequency $f_{b}$, fig. 7 ) is usually sufficient, since this is the critical value: the attenuation due to losses is always less toward the center of the passband (in the case of figure 7, toward infinite frequency) than at the edges.

As an illustration, let us determine the maximum

Table 1. Sample compuiation of dissipation factor

$$
\text { High-pass filter (fig. 7): } \left.\begin{array}{rl}
n_{b} / n_{a} & =\left(f_{a} / f_{b}\right)^{2}=0.612 \\
& n_{c} / n_{a}=0.74 \text { (eq 36) } \\
& n_{b} / n_{c}=n_{b} / n_{a} \cdot n_{a} / n_{c}=\frac{0.612}{0.74}=0.828
\end{array}\right\}
$$

\begin{tabular}{|c|c|c|c|c|}
\hline & Section 1 & Section 2 & Section 3 & Over-all \\
\hline Section number $N=n_{c} / n_{\infty}-2$ & 0 & 0.5 & 0.7 & \\
\hline Ratio $n_{b} / n_{\infty}=\frac{n_{c}}{n_{\infty}} \cdot \frac{n_{b}}{n_{c}}=0.828 \mathrm{~N}$ & 0 & 0.414 & 0.58 & \\
\hline $\begin{array}{l}\text { Real part of } \frac{Z_{s}}{Z_{p}} \text { for } n=n_{b} \text { : } \\
\left.U=\frac{n_{b} / n_{\infty}-n_{b} / n_{c}}{1-n_{b} / n_{\infty}} \text { (eq } 8\right)\end{array}$ & -0.828 & -0.705 & -0.59 & \\
\hline \multicolumn{5}{|c|}{ Assuming $d=0.01$} \\
\hline $\begin{array}{l}\text { Imaginary part of } \frac{Z_{\mathrm{B}}}{Z_{p}} \text { for } n=n_{b} \text { : } \\
V=-d \frac{U}{1-n_{b} / n_{\infty}} \text { (eq 8) }\end{array}$ & 0.00828 & 0.012 & 0.01405 & \\
\hline $\begin{array}{l}\text { Attenuation } \alpha \text {, db: (from chart, } \\
\text { fig. } 8 \text { ) }\end{array}$ & & 0.25 & & \\
\hline $\begin{array}{l}\text { Phase shift } \beta \text {, degrees (from } \\
\text { chart, fig. } 8 \text { ) }\end{array}$ & 131 & 114 & 96 & 341 \\
\hline \multicolumn{5}{|c|}{ Assuming $d=0.02$} \\
\hline$V$ & 0.01656 & 0.024 & 0.0281 & \\
\hline$\alpha$ & 0.32 & 0.46 & 0.48 & 1. 26 \\
\hline$\beta$ & 131 & 114 & 95.5 & 340.5 \\
\hline \multicolumn{5}{|c|}{ Assuming $d=0.0 .5$} \\
\hline$V$ & 0.0414 & 0.06 & 0.0702 & \\
\hline$\alpha$ & 0.96 & 1.15 & 1. 20 & 3. 31 \\
\hline$\beta$ & 130 & 113.5 & 95 & 339.5 \\
\hline
\end{tabular}

The over-all attenuation values thus obtained for several values of $d$ may now be plotted to find, by interpolation, the value of $d$ consistent with the maximum required attenuation at $f_{b}(3 \mathrm{db})$. This is carried out in figure 9, giving as a result $d=0.046$ as the maximum allowable dissipation factor, corresponding to a "Q" of about 22 . 


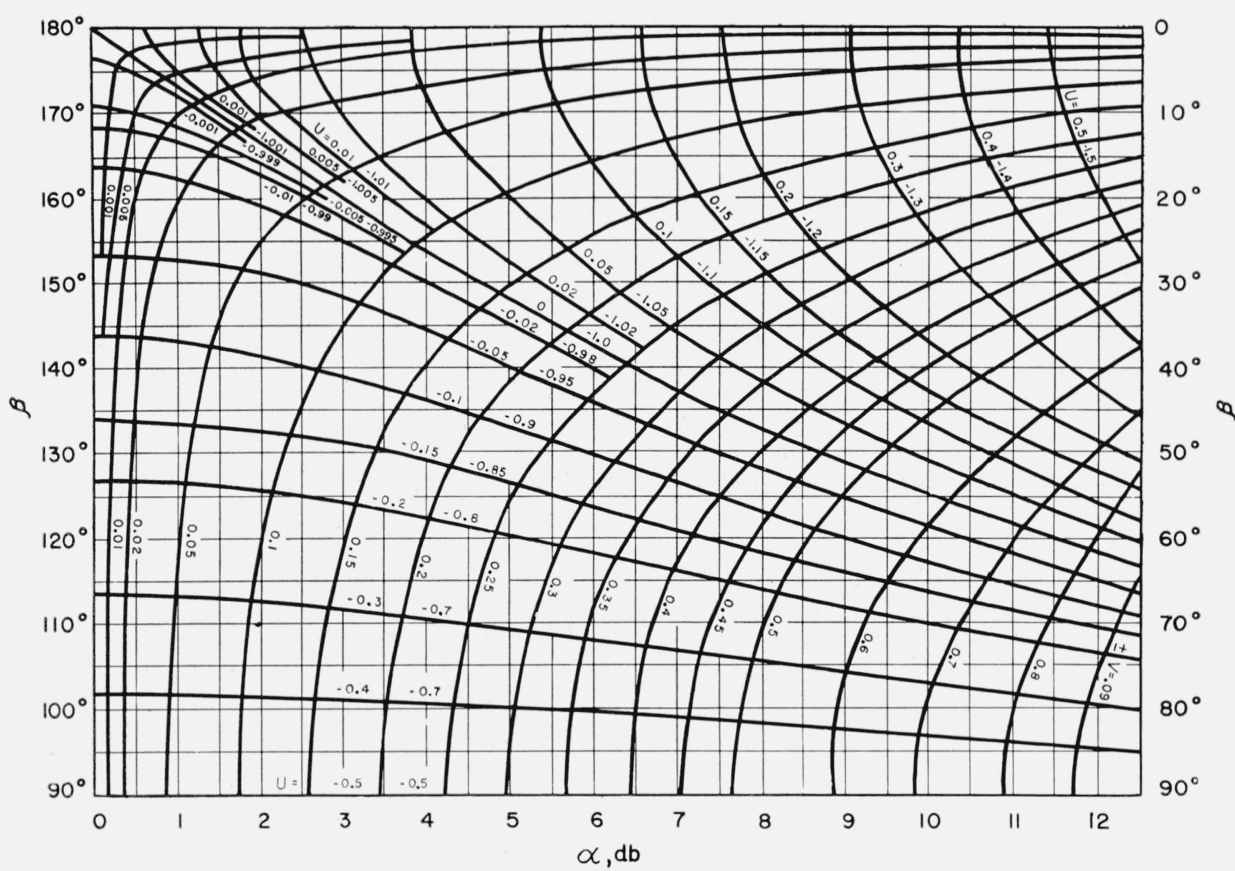

FIGURE 8. Attenuation and phaseshift in term of $U$ and $V$, components of $Z_{s} / Z_{p}$. Note.-For $\mathrm{U}>-0.5,0<\boldsymbol{\beta}<90^{\circ}$; for $U<0.5,90^{\circ}<\boldsymbol{\beta}<180^{\circ}$.

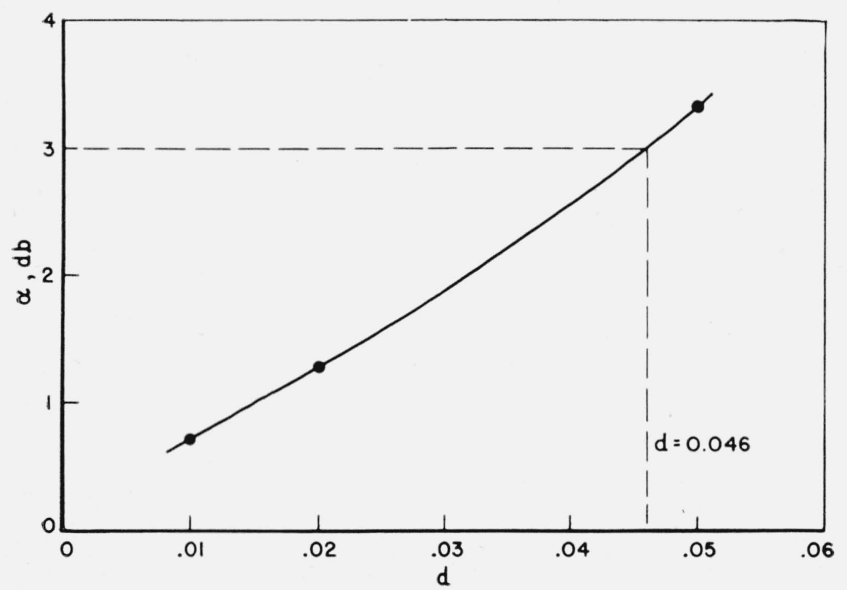

FIGURE 9. Interpolation for maximum dissipation factor.

allowable dissipation (minimum $Q$ ) for the filter of figure 7 , using the values of $N$ already selected.

Equation 8 is used to find $Z_{s} / Z_{p}$ (hence $\alpha$ and $\beta$ ) for each of the three sections of the filter, at the boundary frequency $f_{b}$ (fig. 7 ) and assuming for $d$ (dissipation factor) a series of arbitrary values. Then by interpolation (fig. 9) one may arrive at a value of $d$ just low enough to secure the desired maximum attenuation $\alpha$ (fig. 7) at $f_{b}$. The transition from $Z_{s} / Z_{p}$, as computed from eq 8 , to $\alpha$ and $\beta$, is best accomplished by means of charts, such as that of figure 8. In this chart, as in table 1 , the notation $Z_{s} / Z_{p}=U+j V$ is used. The computation may be conveniently carried out in tabular form, as shown in table 1 .

\section{Midseries and Midshunt Impedances}

Having determined the frequency numbers $n_{c}$ (for the filter) and $n_{\infty}$ (for each filter section) as well as the maximum allowable dissipation factor, the designer still has the problem of "image matching" the filter sections to each other and to the terminations between which the filter is to be inserted. We must, therefore, reexamine the basic filter structures of figure 1 from the standpoint of their image impedances.

In this discussion the $L$ structure, or half-section, has been taken as the basic constituent of the filter. Such a structure has two distinct image impedances: that is, those impedance values which, connected to the input and output of the $L$ network, match, or simulate over the entire frequency range, the input and output impedances of the network, respectively (fig. 11). In filter terminology, the image impedance on the side of the series arm (to the left of fig. $11)$ is called "mid-series impedance", $Z_{0 s}$; the other image impedance is the "mid-shunt impedance", $Z_{0 p}$. The essential difference between the two types of $L$ structures shown in figure 1 under the headings "for connection in $T$ " and "for connection in $\pi$ ", is this: In the first type, the midseries impedance is independent of the resonant frequency of the $L-C \mathrm{arm}$, so that, if such $L$ structures are connected together into $T$ sections, these can be designed to match one another at all frequencies in spite of having different peak attenuation frequencies.

On the other hand, the second type of $L$ structure has midshunt impedance independent of the $L-C$ arm resonance, and must therefore be paired off into $\pi$ sections before assembling into a matching filter. 


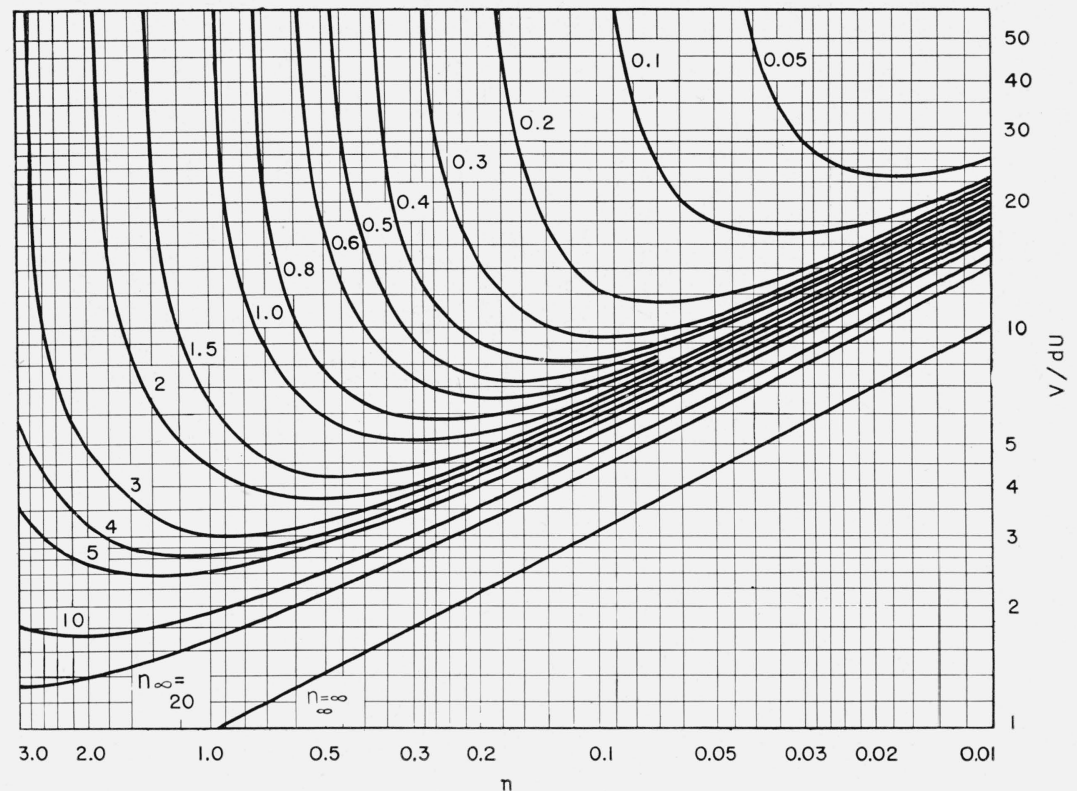

Figure 10. Values of $T$ (imaginary part of $Z_{s} / Z_{p}$ ) in term of $n$ and $n_{\infty}$. To obtain $V$ multiply ordinates by $d$ (dissipation factor) and by $U$ (real part of $Z_{s} / Z_{p}$ ). See eq 45 .

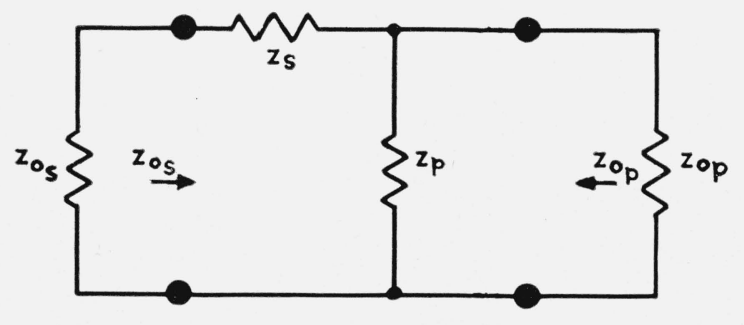

FiguRE 11.

To avoid confusion, let us limit our discussion to the first, or T-connected, type of $L$ structure. The midseries impedance of these will now be obtained, for the nondissipative case.

The midseries impedance is the characteristic impedance of the $T$ (fig. 12). Accordingly, if $Z_{0 c}$ and $Z_{s c}$ are the open and shortcircuited impedances of the $T$, respectively, we have for the mid-series impedance:

$$
\begin{aligned}
Z_{0 s} & =\sqrt{Z_{0 c} Z_{s c}} \\
& =\sqrt{\left(Z_{s}+\frac{Z_{p}}{2}\right)\left(Z_{s}+\frac{1}{2} \frac{Z_{s} Z_{p}}{\left.Z_{s}+\frac{Z_{p}}{2}\right)}\right.} \\
& =Z_{s} \sqrt{1+\frac{Z_{p}}{Z_{s}}}
\end{aligned}
$$

We have already obtained an expression for the arms ratio in the nondissipative case, for all three

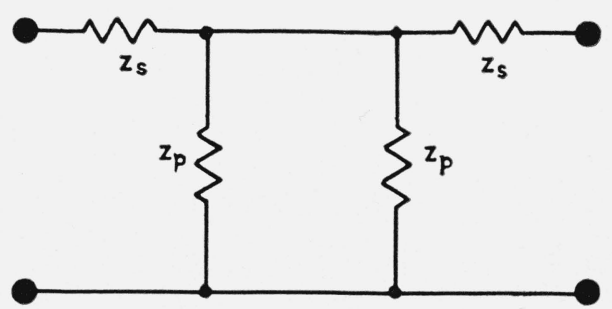

Figure 12.

classes of filters:

$$
\frac{Z_{s}}{Z_{p}}=\frac{\frac{n}{n_{\infty}}-\frac{n}{n_{c}}}{1-\frac{n}{n_{\infty}}} .
$$

We may now substitute this expression into 39 , obtaining:

$$
Z_{0 s}=Z_{s} \sqrt{\frac{\frac{n_{c}}{n}-1}{\frac{n_{c}}{n_{\infty}}-1}} .
$$

We must now differentiate between high-, lowand band-pass filters. For the basic high-pass structure, we have (fig. 3 and eq 4):

$$
Z_{s}=-\frac{j}{\omega C}=-j \frac{\sqrt{n}}{C},
$$


and also (eq 6)

$$
\frac{n_{c}}{n_{\infty}}-1:=-\frac{n_{c}}{C L^{\prime}},
$$

hence combining

$$
Z_{0 s}=-j \sqrt{\frac{L^{\prime}}{C}} \cdot \sqrt{\frac{n}{n_{c}}-1} .
$$

Let us now define:

$$
\text { (high-pass) } \quad R_{0}=\sqrt{\frac{L^{\prime}}{C^{\prime}}},
$$

and write the mid-series impedance in the form:

$$
Z_{0 s}=-j R_{0} \sqrt{\frac{n}{n_{c}}-1}
$$

A plot of $Z_{0 \text { s }}$ against the frequency number $n$ is shown diagrammatically in figure 13. For $n<\eta_{c}$ (transmitted band) the radical must be assigned the positive imaginary value to fit the physical facts: then the midseries impedance is a positive real, with maximum value $R_{0}$ for $n=0$, which corresponds in all cases to the zero phase-shift or "midband" frequency. Hence $R_{0}=\sqrt{L^{\prime} / C}$ is the midband impedance.

It will be noted that $Z_{0 s}$ does not depend upon $n_{\infty}$, hence is not affected by the peak attenuation frequency. High-pass sections differing in the location of the attenuation peak may therefore be image "matched" to one another within the same filter, provided they share the same values of $n_{c}$ (cut-off frequency number) and $R_{0}$. The above plot applies to all three classes of filters except for the sign of the imaginary part; eq 45 also applies, except that $R_{0}$ has different values in terms of the circuit elements. For low-pass filters (see fig. 4 and eq 40):

which can be written

$$
Z_{0 s}=j \omega L \sqrt{\frac{\frac{n_{c}}{n}-1}{\frac{n_{c}}{n_{\infty}}-1}},
$$

$$
Z_{0 s}=j \sqrt{\frac{L}{C^{\prime}}} \sqrt{\frac{n}{n_{c}}-1},
$$

which, if we let

becomes

$$
\sqrt{\frac{L}{C^{\prime}}}=R_{0}
$$

$$
Z_{0 s}=j R_{0} \sqrt{\frac{n}{n_{c}}-1} .
$$

In this case also the midseries impedance becomes a positive real in the range $n<n_{c}$, provided we assign a negative imaginary value to the radical. Outside this frequency range the impedance is a positive reactance, whose dependence on $n$ is the same as in the case of high-pass filters, except for sign (fig. 13).

Finally, for symmetrical band-pass filters (fig. 5, eq 40)

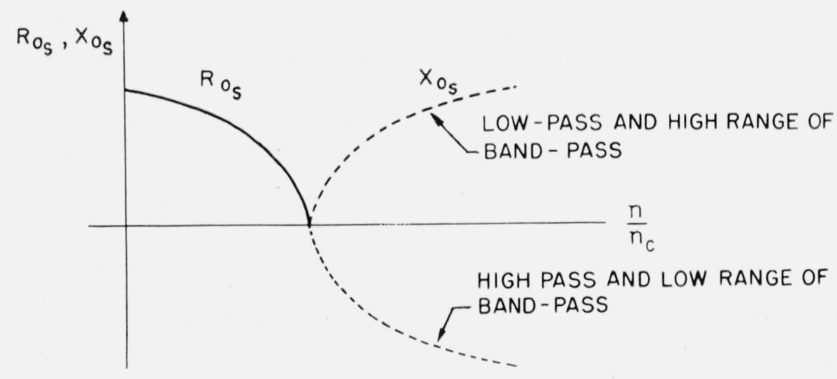

Figure 13.

$$
\begin{aligned}
Z_{0 s} & = \pm j r \sqrt{n} \sqrt{\frac{n_{c} / n-1}{n_{c} / n_{\infty}-1}} \\
& = \pm j r \frac{\sqrt{n / n_{c}-1}}{\sqrt{1 / n_{c}-1 / n_{\infty}}} .
\end{aligned}
$$

The midseries impedance will have the same form as in the high-pass and low-pass cases, provided:

$$
r=R_{0} \sqrt{\frac{1}{n_{c}}-\frac{1}{n_{\infty}}},
$$

in which case:

$$
Z_{0 s}= \pm j R_{0} \sqrt{\frac{n}{n_{c}}-1} .
$$

$R_{0}$, as before, is the midband impedance. The midseries impedance follows the plot of figure 13 for $n>n_{c}$; the sign of $\sqrt{n}$ in eq 49 is positive if the frequency is above the transmitted band ( $Z_{0 s}$ inductive), and negative if below.

In conclusion, we have found that in all three cases (high, low, and band-pass) the midseries impedance is the same function of the frequency number; in each case it is, therefore, invariant throughout the filter, whatever the location of the attenuation peaks in the individual sections. This permits the filter to be image-connected throughout; which is the assumption upon which the entire "conventional" filter theory is predicated. Of course, this is only true provided $R_{0}$, the midband impedance, and $n_{c}$, the cut-off frequency number, are constant throughout the filter. 'This is ensured by determining these two parameters first and selecting element values so that eq 44, 48 and 51, for high, low and band-pass filters, respectively, are satisfied.

We have discussed the determination of $n_{c}$ but not that of $R_{0}$. This is based on the terminating impedance of the filter. Always assuming that the "T-connected" type of structure is used, and therefore, that the midseries impedance is invariant from section to section, we could put together the filter (a three-section filter, for example) as indicated in figure 14. Each $L$ half-section is shown separate: in practice, adjoining arms are "lumped" together.

This arrangement would offer to the outside terminations the same impedance (the midseries impedance) that recurs at each internal junction. If the terminations are equal resistances, which we 
SOURCE

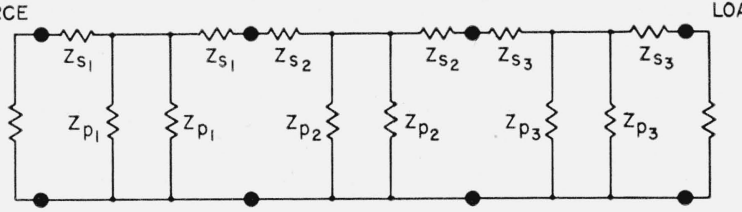

Figure 14.

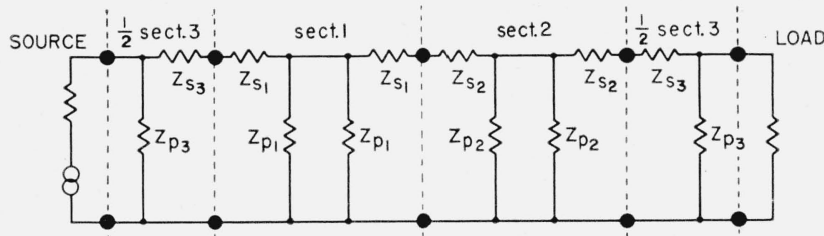

Figure 15.

assume to be the case, considerable "mismatching" would result, as indicated by the plot of figure 13; the midseries impedance plot is actually an arc of circle within the transmitted range when frequency, rather than $n$, is taken as the abscissa.

\section{Terminating Half-sections}

A significant improvement may result if we connect the filter as shown in figure 15. One of the sections, suitably selected, is "split" into its component halfsections and these are used to "terminate" the filter. The filter now presents to its terminations (source and load) not the midseries, but the midshunt impedance of the terminating section.

The frequency dependence of the midshunt impedance must now be examined. This will show how the terminating section should be selected and how the parameter $R_{\theta}$, equal to the midband value of both the midshunt and the midseries impedances, should be assigned with reference to the outside terminations.

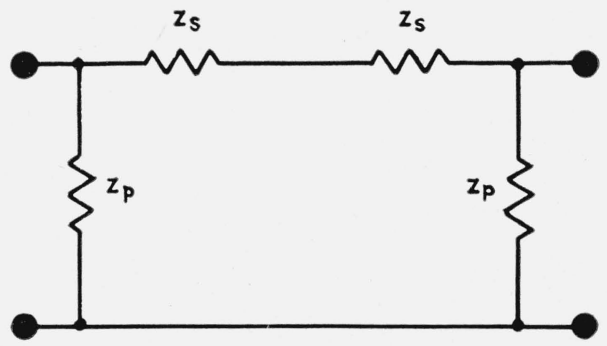

Figure 16.

The midshunt impedance $Z_{0 p}$ is the characteristic impedance of the $\pi$ section of figure 16 . We have, therefore:

$$
\begin{aligned}
Z_{0 p}= & \sqrt{Z_{0 c} Z_{s c}} \\
= & \frac{Z_{p}}{\sqrt{1+\frac{Z_{p}}{Z_{s}}}}=\frac{Z_{p} Z_{s}}{Z_{0 s}}=\frac{Z_{p}}{Z_{s}}\left(\frac{Z_{s}}{Z_{0 s}}\right)^{2} Z_{0 s}
\end{aligned}
$$

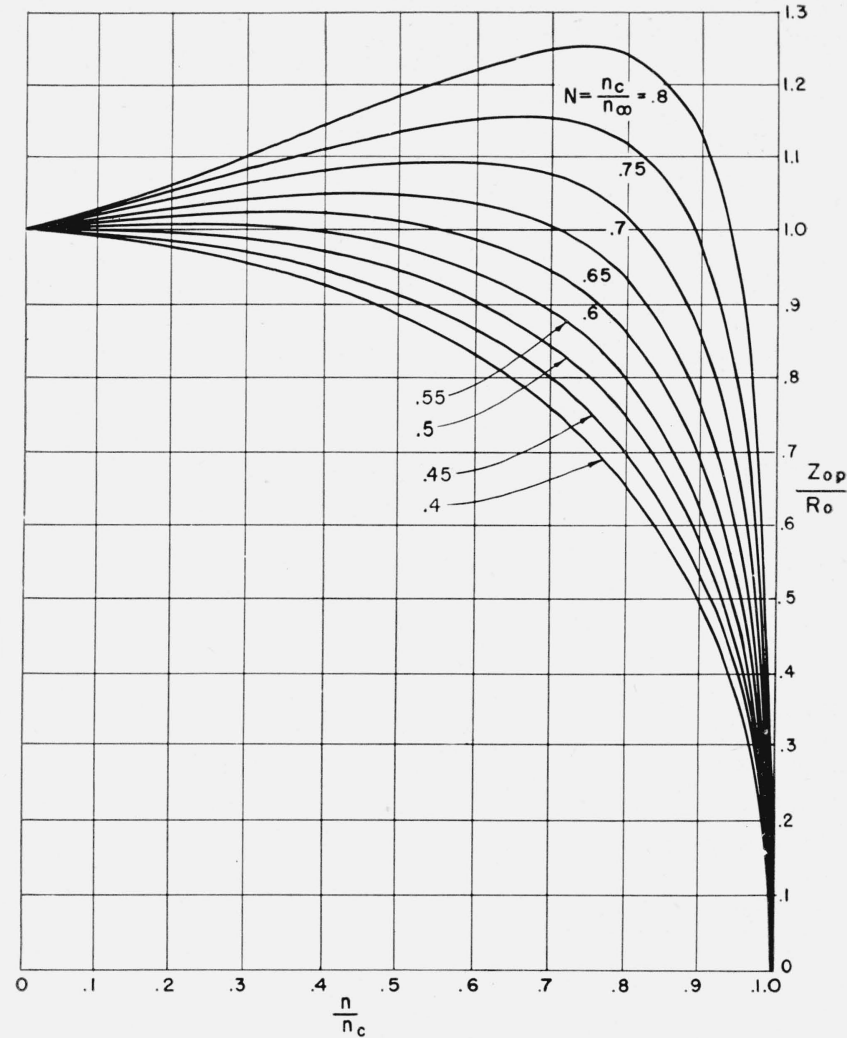

Figure 17. Plots of $\eta_{0 p} / R_{0}$ against $n / n_{c}$.

Using eq 30,45 , and 52, we may write:

$$
Z_{0 p}= \pm j R_{0} \frac{1-\frac{n}{n_{\infty}}}{\sqrt{\frac{n}{n_{c}}-1}} .
$$

The midshunt impedance, like the midseries, is real in the transmission range $\left(n<n_{c}\right)$ and takes the value $R_{0}$ at midband $(n=0)$. Its frequencydependence, however, may vary from section to section according to the value of $n_{\infty}$. Plots of $Z_{0 p}$ against $n / n_{c}$ in the transmitted band are given in figure 17. These show that for $N=n_{c} / n_{\infty}=0.7$, or thereabouts, $Z_{0 p}$ is substantially constant over a large part of the transmitted band. This is the value of $N$ that should be used in designing the terminating section.

\section{Determination of $\mathrm{R}_{0}$}

The use of properly designed terminating sections reduces, but does not eliminate, reflection losses due to mismatching at the terminations [1, p. 352ff]. These losses can be evaluated, but if the designer merely has to meet some such requirement as that shown in figure 7 , this is not necessary. If the filter is designed to match its terminations exactly at $f=f_{b}$, at this frequency there will be no added loss due to mismatching. Above and below this frequency there may be some added loss, but attenua- 
tion falls off toward midband more rapidly than would be required to offset the increasing reflection loss.

The high-pass filter previously chosen as example, and based upon the requirements of figure 7 , includes a section for which $N=0.7$ (section 3 ). Choosing this as the terminating section, we go to the plots of figure 17. The abscissa corresponding to $f=f_{b}$ is (eq 38 , table 1$)$ :

$$
\frac{n_{b}}{n_{c}}=0.828
$$

Hence, in order to avoid mismatching, we must have, if $R_{T}$ is the terminating resistance:

$$
Z_{0 s}=R_{T},
$$

at the point where the curve for $N=0.7$ crosses the abscissa $n / n_{c}=0.828$.

But at this point:

$$
\frac{Z_{0 s}}{R_{0}}=0.985
$$

hence, we must make:

$$
R_{0}=\frac{R_{T}}{0.985}=1.015 R_{T}
$$

The difference between the optimum value of $R_{0}$ and that of $R_{T}$ is generally small, so that $R_{0}$ is often taken equal to $R_{T}$. But this simplification is not always permissible if accuracy is desired.

\section{Computation of Filter Elements}

From the design parameters $n_{c}$ and $R_{0}$, common to all the sections of a filter, and $N=n_{c} / n_{\infty}$, varying from section to section, plus, in the case of symmetrical band-pass filters, the midband angular frequency $\omega_{0}$, all the filter element values may be obtained by straightforward computation. Table 2 gives the expressions for all element values of the three "basic" structures of the "T-connected" type, in terms of the parameters used throughout this discussion, and with reference to equations appearing elsewhere in the discussion. Because of the dual relationship between "T-connected" " $\pi$-connected" filters, it is easy to obtain the equivalent $\pi$-connected expressions: as always in a dual circuit relationship, capacitance is replaced by inductance, resistance by conductance, series by parallel, and vice-versa $[1$, p. $246 \mathrm{ff}]$.

The so-called "prototype" sections are considered here as limiting cases of the "basic" section, for $N=0$. In high- and low-pass sections, the element values are obtained in this limiting case by simply making $N=0$ in the expressions for the basic section. One of the elements drops out, as indicated by the fact that the capacitor $C^{\prime}$ in the high-pass section becomes infinite and the inductor $L^{\prime}$ in the low-pass section goes to zero. In the band-pass case, it is not convenient to let $N$ go to zero, as indeterminate expressions will result. Separate expressions for the prototype elements, which can be obtained quite simply by going back to basic considerations, are given.

Finally, it should be noted that the series arms of adjoining sections are combined into a single arm of the assembled filter. Thus, the series inductances add in the low-pass case and the series capacitances go together according to the usual rules in the highpass case. In band-pass filters, it is convenient to add together values of $r=\sqrt{L / C}$ for adjoining sections. This will give the value of $r$ for the "combined" series arm. As for the resonant frequency, this is the same for all series arms, hence also for their combinations.

\section{Conclusion}

Comparison with conventional method. The principal difference between the method outlined here and the "classical" method lies in the choice of the design parameters for the simply derived filter section. In the classical method these are: $f_{c}$, cut-off frequency, and $m$ derivation factor. In the modified method they are: $n_{c}$, value of the frequency number at cut-off, and $n_{\infty}$, value of the frequency number at the frequency of peak attenuation. Why is this an advantage? Because the frequency number is that particular variable that permits the use of a single function, or a single family of curves, to express the attenuation and phase-shift characteristics of all filter sections (low, high, and band-pass) over the frequency spectrum. With the aid of these universal curves or the corresponding functions it is simple to relate the design parameters, as used in the present method, with performance. If any other set of parameters is used, an intermediate step is required.

When using the classical method the designer is required to choose values of $f_{c}$ and $m$ based upon his general knowledge of filter characteristics. When the problem is an academic one $f_{c}$ is usually given, but this is not the case in practice, the requirements of the filter being summarized in some form similar to figure 7 . After $f_{c}$ and $m$ have been selected, formulas are given for obtaining $f_{\infty}$, the peak attenuation frequency; on the basis of this and $f_{c}$ the filter performance can be checked, to see whether or not the choice of $f_{c}$ and $m$ was correct in the first place. With the present method the choice of parameters and the verification of performance are one and the same. The parameters are selected by means of the universal charts, which show attenuation and phaseshift at the various frequencies.

An additional improvement made possible by the present method is the computation of "minimum $Q$." The new parameters greatly simplify the analysis of dissipative filter sections. Formulas given in classical texts for the dissipative value of $Z_{s} / Z_{p}$ in the band-pass case, for example, are much more complicated than eq 29 and do not permit the drawing of a single family of curves as in figure 10 . 
TABLE 2. Computation of element values

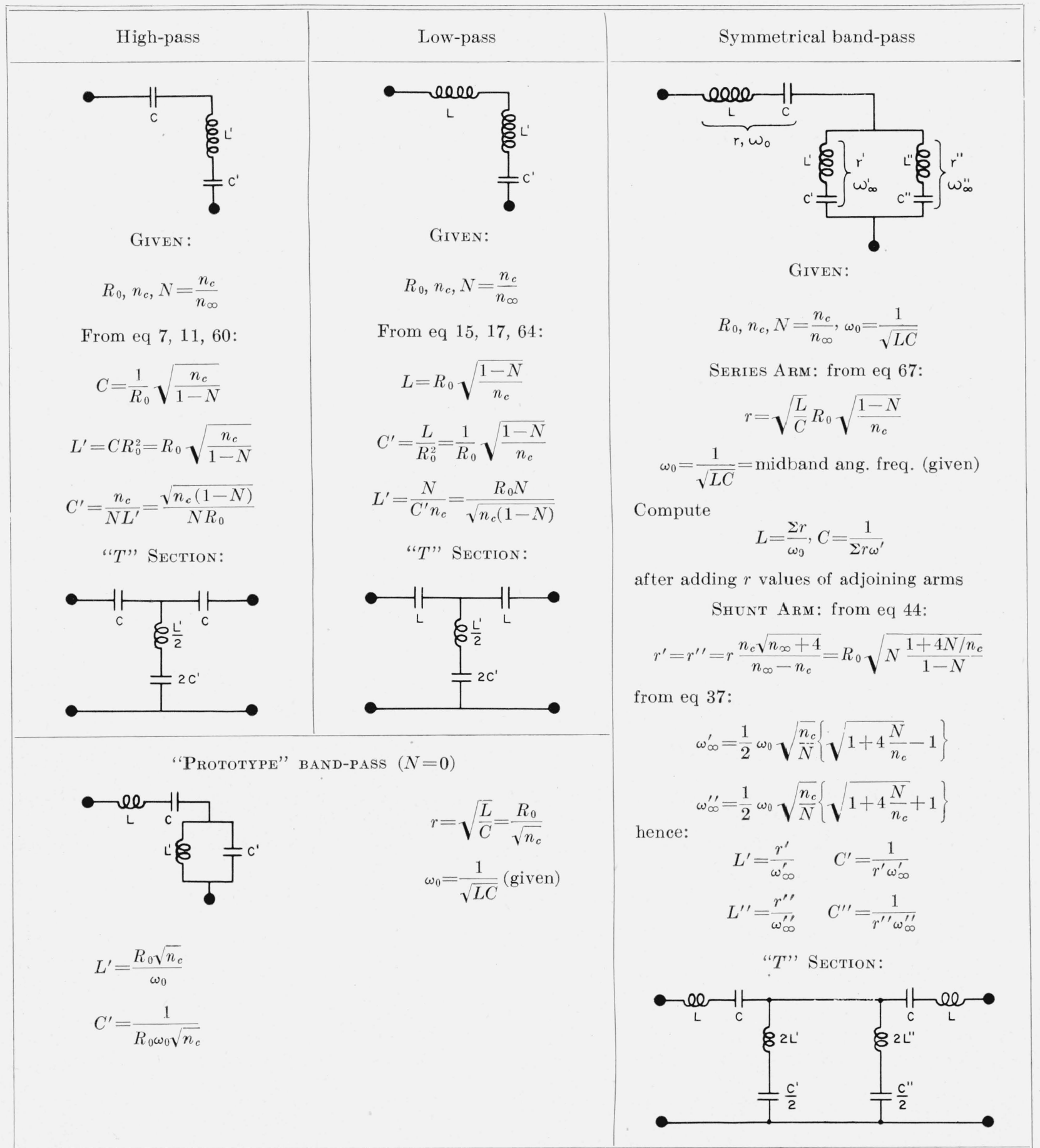

\section{References}

[1] E. A. Guillemin, Communication networks, Vol. II, p. $378 f f$ (John Wiley \& Sons, Inc., New York, N. Y., 1948).

[2] O. J. Zobel, Theory and design of uniform and composite electric wave filters, Bell System Tech. J. (Jan. 1923).

[3] W. Cauer, New theory and design of wave filters, Phys. (April 1932).

[4] H. W. Bode, A general theory of electric wave filters, J. Math. \& Phys. (Nov. 1934).
[5] P. J. Selgin, Electrical transmission in steady state, p. $47 \mathrm{ff}$ (McGraw-Hill Book Co., Inc., New York, N. Y., 1946).

[6] T. E. Shea, Transmission networks and wave filters (D. Van Nostrand \& Co., Inc., New York, N. Y., 1948).

[7] P. J. Selgin, Multi-section filter design procedure, Communications (July 1945).

Washington, February 7, 1950. 